\title{
Complications and Costs Associated with Initiation of Anti-coagulation Therapy Using Warfarin-An Observation Study
}

Jasmine Chiat Ling Ong ${ }^{1}$, Seng Han Lim¹ , Chai Ling Ong², Liping Chen², Elicia Purnata², Edmund Joo Sheng See $^{1}$, Joel Cho-Lun Chiang ${ }^{1}$, Vivian Shi Yin Wong ${ }^{1}$, Loke Khee Siow ${ }^{1}$, Jin Shing Hon $^{2}$, Ming Chai Kong ${ }^{1}$ and Chi Keong Ching ${ }^{3}$

1. Department of Pharmacy, Singapore General Hospital, Outram Road, Singapore 169608, Singapore

2. Department of Pharmacy, National Heart Centre Singapore, 5 Hospital Drive, Singapore 16960, Singapore

3. Department of Cardiology, National Heart Centre Singapore, 5 Hospital Drive, Singapore 169609, Singapore

\begin{abstract}
To evaluate the safety and cost of anticoagulation therapy in patients newly initiated on warfarin in two institutions in Singapore. All patients newly started on warfarin between December 2011 and May 2012 were recruited and followed up for a period of 6 months. All hospitalization attributed to complications of warfarin therapy, number of INR (international normalized ratio) greater than 4 and total cost of warfarin therapy were collected and analysed. A total of 321 patients were newly initiated on warfarin for standard indications were recruited. At the end of 6 month period, 8.4\% of patients were hospitalized for complication related to warfarin therapy. For patients who had stable INR while on warfarin, this rate was almost 2 times lower (3.8\% vs $8.4 \%$ ). In our study group, patients started on warfarin as inpatients had higher rates of bleeding (15.1\% vs 6.7\%) and more INRs greater than 4 (0.7 per patient vs 0.11 per patient). The cost of initating warfarin was USD660 over the first 6 months of therapy. Patients newly initiated on warfarin were at a heightened risk of bleeding complications as compared to patients already stable on warfarin therapy. This also translates to considerable costs of warfarin initiation.
\end{abstract}

Key words: Anticoagulation, warfarin, initiation, complications, cost.

\section{Introduction}

The use of warfarin remains prevalent after the introduction of novel anticoagulatants. In a national survey conducted in the United Sates of America, warfarin was identified as one of top three drugs resulting in emergency department visits. Amongst cases attributed to warfarin, $73 \%$ involved clinically evident bleeding and $44.2 \%$ of patients required hospitalization [1]. A large integrated health care system in Northern California studied 11526 patients with nonvalvular atrial fibrillation on warfarin reported an incidence of intracranial hemorrhage twice

Corresponding author: Chi Keong Ching, MBBS, MRCP (UK), FAMS, associate professor, research field: eectrophysiology and pacing. E-mail: ching.chi.keong@nhcs.com.sg. as high compared to patients not on warfarin [3]. This large community of patients on warfarin continues to require close monitoring in view of the drug's narrow therapeutic index [2]. The safety of warfarin therapy can be improved by introducing safety measures, e.g. managing patients in specialized anticoagulation clinics, introducing guidelines and protocols for warfarin initiation, setting up inpatient anticoagulation teams [3]. The healthcare outcomes of patients improve further in hospitals with pharmacist-provided anticoagulation management in terms of less bleeding complications, deaths, blood transfusions required among other benefits, in contrast to hospitals without pharmacist-provided warfarin therapy management [4]. Specialized anticoagulation service is available at ambulatory clinics in the NHCS (National Heart 
Centre Singapore), a specialist centre for cardiac diseases and the SGH (Singapore general hospital), a tertiary hospital in Singapore. Till date, there are no studies exploring the safety warfarin therapy during the initiation phase in an asian context. The aim of this project is to study the incidence of adverse events in patients newly initiated on warfarin.

\section{Methods}

\subsection{Study Design and Sample}

This is a retrospective study of warfarin naïve patients, newly initiated on warfarin and followed up at the outpatient clinics in two centers of the Outram Campus Singapore. The Outram Campus Singapore consists of a national specialist centre, NHCS (national heart centre Singapore) and a large local tertiary institution, SGH (Singapore general hospital).

Electronic medical and pharmacy records between the period of 1st December 2011 and 31st May 2012 were screened. Patients who were warfarin naïve, newly initiated on warfarin for labelled indications of warfarin for example AF (atrial fibrillation) and DVT (deep vein thrombosis) and followed up within Outram Campus Singapore, by either NHCS or SGH for at least 6 months after initiation or until completion of therapy, whichever earlier. Patients with index warfarin prescription before the study period or lost to follow up were excluded from the study. Warfarin initiation dates for each patient were cross-confirmed with the respective electronic medical records for accuracy. Included patients were followed up for a period of 6 months from the time of warfarin initiation for the primary and secondary outcomes of the study.

Prescription and other medical records were obtained from the institution's computerized physician order entry system (sunrise clinical manager), while pharmacy refills records were extracted from electronic dispensing system (Maxcare). This study was granted exemption from the ethics committee by the Institution Review Board of Singhealth,
Singapore.

\subsection{Outcome Measures}

The primary outcome of this study was 6 months rehospitalisation rate for bleeding or thrombosis attributed to warfarin therapy. Bleeding events were classified as major or minor bleeding as defined by The International Society on Thrombosis and Haemostasis [5].

Secondary outcome of this study was the economic burden to initiate warfarin therapy in these patients during the first 6 months of therapy. Economic burden included the direct cost incurred due to number of actionable INR (defined as INR $>4$ ); number of outpatient visits (consultation and laboratory fees) and re-admission due to complications of warfarin therapy.

\subsection{Data Collection and Statistical Analysis}

Clinical demographics collected in this study were age, gender, ethnicity and indication for warfarin. Age was recorded as a continuous variable, while gender and ethnicity were reported as categorical variables. Other variables included number of re-admissions due to complications of warfarin therapy, number of INR values $>4$, mortality attributed to warfarin therapy and all cause mortality. Descriptive statistics were used for both the primary and secondary outcomes.

\section{Results}

\subsection{Patient Demographics (Table 1)}

A total of 321 patients were newly initiated on warfarin within the study period of 1st December 2011 to 31st May 2012. Out of these 321 patients, $62.6 \%$ were males. The mean age of patients newly initiated on warfarin was $63.4 \pm 13$ years old, with the females averaging 66.3 years old and males averaging 61.6 years old. In terms of ethnicity distribution, the distribution follows the general ethicity proportion of the Singapore population, with Chinese as the majority (76.0\%), followed by Malay (12.8\%), Indian (3.4\%) and Eurasian (1.2\%). Other ethinicities made 
Table 1 Patient demographics and indications of warfarin.

\begin{tabular}{lll}
\hline \multicolumn{3}{l}{ Patient demographics and indications of warfarin } \\
\hline Gender & $\mathrm{n}$ & \multicolumn{1}{l}{$(\%)$, unless otherwise stated } \\
Female & 120 & 37.4 \\
Male & 201 & 62.6 \\
Age & & \\
Years of age & 63 & $\pm 13(\mathrm{SD})$ \\
Race & & \\
Chinese & 244 & 76 \\
Malay & 41 & 12.8 \\
Indian & 11 & 3.4 \\
Eurasian & 4 & 1.2 \\
Others & 21 & 6.5 \\
Indication & & \\
AF & 195 & 60.7 \\
DVT & 40 & 12.5 \\
PE & 27 & 8.4 \\
LV thrombus & 22 & 6.9 \\
MVR & 14 & 4.4 \\
AVR & 10 & 3.1 \\
Others & 13 & 4 \\
\hline
\end{tabular}

up the remaining $6.5 \%$ of the study population.

Patients were started on warfarin for the following indications: atrial fibrillation (60.7\%), followed by deep vein thrombosis (12.5\%) and then pulmonary embolism (8.4\%), left ventricular thrombus (6.9\%), mechanical mitral/aortic valve replacement (7.5\%).

\subsection{Primary Outcome: Re-admission Due to Complications of Warfarin Therapy (Table 2)}

Patients newly initiated on warfarin were followed up for a period of 6 months into their warfarin therapy to determine the rate of re-admission due to complications of warfarin therapy, i.e. major bleeding, minor bleeding, thrombosis [5] or high INR which required hospital admission for monitoring as decided by their physician. Within 6 months of warfarin therapy, there were 27 (8.4\%) unique incidences of re-admission due to complications of warfarin therapy. Out of which, 3 incidences were classified as major bleeding and 8 incidences as minor bleeding. The rest of the re-admissions were due to high INR which required hospital admission for monitoring. There were no incidence of thrombosis due to subtherapeutic warfarin therapy.

More than $20 \%$ of patients had at least an episode of INR greater than 4 during the 6 months intiation of warfarin therapy. Total number of INR greater than 4 for all patients within the 6 months initiation of warfarin therapy was 103 . Within 6 months initiation of warfarin therapy, 14 patients died, with 4 fatalities directly attributed to complications of warfarin therapy.

In our study group, patients started on warfarin as inpatients had higher rates of bleeding $(15.1 \%$ vs $6.7 \%)$ and more INRs greater than 4 (0.7 per patient vs 0.11 per patient).

\subsection{Secondary Outcome: Economic Burden within 6 Months of Initiating Warfarin Therapy (Table 3)}

Both inpatient and outpatient cost incurred by the patients due to warfarin therapy were taken into consideration for the economic burden within 6 months of intiating warfarin therapy. On average, one incidence of re-admission due to complications of warfarin therapy cost approximately USD3760. The inpatient cost over 6 months of initiating warfarin therapy was approximately USD320 per patient. Outpatient cost consisted of laboratory fees (capilllary INR) and physicians' consultation fees over 6 months. A total of approximately USD340 per patient is incurred over 6 months (including both the laboratory and consultation fees) for outpatient consultations. This

Table 2 Clinical events due to complication of warfarin therapy.

\begin{tabular}{lll}
\hline Clinical events due to complication of warfarin therapy & \\
\hline & $\mathrm{n}$ & $(\%)$ \\
Major bleeding & 3 & 0.9 \\
Minor bleeding & 8 & 2.5 \\
Thrombosis & 0 & 0.0 \\
Total hospitalisation due to warfarin therapy & 27 & 8.4 \\
No. of INR $>4$ & 103 & - \\
No. of patients with $>=1$ episode of INR $>4$ & 77 & 24.0 \\
Mortality due to warfarin therapy & 4 & 1.2 \\
All-cause mortality & 14 & 4.1 \\
\hline
\end{tabular}


Table 3 Economic burden within 6 months of initiating warfarin therapy.

\begin{tabular}{lll}
\hline Economic burden within 6 months of initiating warfarin therapy & & S.D. \\
\hline & & in USD \\
Inpatient cost (Out of 27 events) & $\$ 3,760.00$ & $\$ 6,040.00$ \\
Due to complications of warfarin therapy (Per event) & $\$ 320.00$ & $\$ 1,990.00$ \\
Due to complications of warfarin therapy (Per patient) & & $\$ 70.00$ \\
Outpatient cost per patient & $\$ 90.00$ & $\$ 200.00$ \\
Laboratory cost per patient & $\$ 250.00$ & $\$ 660.00$ \\
Consultation cost per patient & & \\
Total cost per patient & & \\
\hline
\end{tabular}

Table 4 Reasons for discontinuation of warfarin.

\begin{tabular}{ll}
\hline Reasons for discontinuation of warfarin & \\
\hline Total discontinuation & 41 \\
Changed to NoACs & 14 \\
Changed to antiplatelets & 16 \\
Completely stopped & 11 \\
Stopped due to labile INR & 18 \\
Stopped due to bleeding incident & 4 \\
\hline
\end{tabular}

included approximately USD90 and USD250 for all laboratory fees and consultation fees respectively, over 6 months of initiating warfarin therapy for one patient. Taking all cost in total, an average warfarin naïve patient newly initiated on warfarin for a 6 months period will incur approximately USD660.

\subsection{Secondary Outcome: Reasons for Discontinuation of Warfarin (Table 4)}

A total of 41 patients (21.8\%) discontinued use of warfarin during the period of follow up. The main reason for discontinuation was labile INR. These patients were subsequently started on dual antiplatelet therapy or new oral anticoagulants including rivaroxaban and dabigatran.

\section{Discussion}

Unlike most other studies which focused only on patients taking warfarin for atrial fibrillation [6-8], our study included all patients with standard indications of warfarin (e.g. DVT, PE, AF and LV thrombus). Previous reports had suggested a higher risk of bleeding and thrombosis during initial warfarin therapy, as compared chronic warfarin therapy [9-10].
Our study is the first to report results on new warfarin patients based on a Southeast Asian population, providing a different ethnic perspective from studies investigating Caucasian populations. However, as compared to many other economic studies on warfarin therapy, our study took on a patient-oriented approach and did not take into account cost incurred by the healthcare institution.

In contrary to common belief that warfarin therapy is cheap due to the low cost of warfarin tablet, this study highlighted that there is significant cost incurred to patients especially during the initiation phase of therapy, when monitoring of the efficacy and safety is expected to be more intense.

Our study highlighted that an average of USD660 was incurred per patient for outpatient warfarin therapy and inpatient hospitalisation fees over a period of 6 months from the time of warfarin initiation. In a study conducted by Hallinen et al. [11], 263 euros was incurred per patient during a 60-day warfarin initiation period. This cost incurred parallels the cost incurred by our patients. Several other papers have also attempted to quantify the cost of warfarin therapy. In a retrospective study analyzing the cost of anticoagulation in AF patients, Walsh et. al. [12] reported both the costs incurred by a warfarin patient and also the cost incurred by the healthcare institution. The results demonstrated that despite the low cost of the drug, total cost per patient per visit was 70.07 euros, which included costs to the health care provider per patient visit of 21.57Euros and cost incurred by patient per visit of 48.50Euros. The authors concluded 
that despite low drug cost, the cost of patient monitoring is significant. On a different note, the cost of warfarin therapy for stable patients can be comparatively lower that incurred during the initiation phase. In an observational conducted in the United Kingdom, total cost of warfarin treatment was estimated to be USD250 per patient per year (mean follow up period of 19 months) [13]. This is a stark difference from our results, demonstrating a need to reduce cost of warfarin initiation.

In comparison to NOACs (new oral anticoagulants) such as Factor Xa inhibitor or direct thrombin inhibitor, warfarin had frequently been shown to be less cost effective. Dabigatran for one, was shown by Chang et. al. to be highly cost-effective compared to warfarin in a Hong Kong based teaching hospital [14]. Similar results were concluded in Taiwan. Most of these studies were, however, conducted in a caucasian population and may not be truly reflective of an asian population like Singapore.

In our study, the re-admission rate due to bleeding attributed to warfarin therapy was $8.4 \%$. This figure worked out to be 16.8 incidences of bleeding per 100 patient years. This figure is higher than the re-admission rate of 3.8 incidences of all bleeding per 100 patient years as concluded by Gallagher et. al. [15]. The main difference in the incidences of bleeding could have been due to our study including only warfarin naïve patients, newly initiated on warfarin therapy for 6 months. In their study all patients currently on warfarin therapy were included. This once again re-emphasised the fact that newly initiated patients were at higher risk of bleeding complications from warfarin therapy, as summarised by Garcia et.al. [16].

Since a huge amount of cost incurred to patients on warfarin therapy comes from hospitalisation bills, it was intuitive that patients newly initiated on warfarin therapy would therefore incur a higher cost of therapy, as compared to patients who were on warfarin therapy for some time.

\section{Conclusion}

In warfarin-naïve patients, newly initiated on warfarin therapy, the rate of re-admission due to complications of warfarin therapy was $8.4 \%$ for a 6 months follow up period or 16.8 incidences per 100 patient years. On average, a warfarin patient pays USD660 for a period of 6 months from the time of initiation, and much higher if the patient develops complications.

\section{References}

[1] Budnitz, D. S., Shehab, N., Kegler, S. R., and Richards, C. L. 2007. "Medication Use Leading to Emergency Department Visits for Adverse Drug Events in Older Adults.” Ann. Intern. Med. 147 (11): 755-65.

[2] Ageno, W., Gallus, A. S., Wittkowsky, A., Crowther, M., Hylek, E. M. and Palareti, G. 2012. "Oral Anticoagulant Therapy: Antithrombotic Therapy and Prevention of Thrombosis, 9th ed: American College of Chest Physicians Evidence-Based Clinical Practice Guidelines.” Chest 141: e44S-88S.

[3] Go, A. S., Hylek, E. M., Chang, Y. C., Phillips, K. A., Henault, L. E., and Capra, A. M. 2003. Anticoagulation Therapy for Stroke Prevention in Atrial Fibrillation-How Well Do Randomised Trials Translate into Clinical Practice?” JAMA 290: 2685-92.

[4] Bond, C. A., and Raehl, C. L. 2004. "Pharmacist-Provided Anticoagulation Management in United States Hospitals: Death Rates, Length of Stay, Medicare Charges, Bleeding Complications, and Transfusions.” Pharmacotherapy 24 (8): 953-63.

[5] Schulman, S., and Kearon, C. 2005. "Subcommittee on Control of Anticoagulation of the Scientific and Standardization Committee of the International Society on Thrombosis and Haemostasis, Definition of Major Bleeding in Clinical Investigations of Antihemostatic Medicinal Products in Non-surgical Patients.” J. Thromb Haemost 3 (4): 692-4.

[6] Azoulay, L., Dell’Aniello, S., Simon, T. A., Renoux, C., and Suissa, S. 2014. "Initiation of Warfarin in Patients with Atrial Fibrillation: Early Effects on Ischaemic Strokes.” Eur. Heart J. 35 (28): 1881-7.

[7] Jacobs, L. G., Billett, H. H., Freeman, K., Dinglas, C., and Jumaquio, L. 2009. "Anticoagulation for Stroke Prevention in Elderly Patients with Atrial Fibrillation, Including Those with Falls and/or Early-Stage Dementia: A Single-Center, Retrospective, Observational Study.” Am. J. Geriatr. Pharmacother 7 (3): 159-66. 
[8] McCormick, D., Gurwitz, J. H., Goldberg, R. J., Becker, R., Tate, J. P., Elwell, A., and Radford, M. J. 2001. "Prevalence and Quality of Warfarin Use for Patients with Atrial Fibrillation in the Long-Term Care Setting.” Arch. Intern. Med. 161 (20): 2458-63.

[9] Gomes, T., Mamdani, M. M., Holbrook, A. M., Paterson, J. M., Hellings, C., and Juurlink, D. N. 2013. "Rates of Hemorrhage during Warfarin Therapy for Atrial Fibrillation.” CMAJ 185 (2): 121-7.

[10] Hylek, E. M., Evans-Molina, C., Shea, C., Henault, L. E., and Regan, S. 2007. "Major Hemorrhage and Tolerability of Warfarin in the First Year of Therapy among Elderly Patients with Atrial Fibrillation.” Circulation 115 (21): 2689-96.

[11] Hallinen, T., Martikainen, J. A., Soini, E. J., Suominen, L., and Aronkytö, T. 2006. "Direct Costs of Warfarin Treatment among Patients with Atrial Fibrillation in a Finnish Health Care Setting." Curr. Med. Res. Opin. 22 (4): 683-92.

[12] Walsh, C., Murphy, A., Kirby, A., and Vaughan, C. 2014.
“Retrospective Costing of Warfarin.” Ir. Med. J. 107 (5): 133-5.

[13] Abdelhafiz, A. H., and Wheeldon, N. M. 2003. "Use of Resources and Cost Implications of Stroke Prophylaxis with Warfarin for Patients with Nonvalvular Atrial Fibrillation.” Am. J. Geriatr. Pharmacother 1 (2): 53-60.

[14] Chang, C. H., Yang, Y. H., Chen, J. H., and Lin, L. J. 2014. "Cost-Effectiveness of Dabigatran Etexilate for the Prevention of Stroke and Systemic Embolism in Atrial Fibrillation in Taiwan. Thromb Res. 133 (5): 782-9.

[15] Gallagher, A. M., van-Staa, T. P., Murray-Thomas, T., Schoof, N., Clemens, A., Ackermann, D., and Bartels, D. B. 2014. "Population-Based Cohort Study of Warfarin-Treated Patients with Atrial Fibrillation: Incidence of Cardiovascular and Bleeding Outcomes.” BMJ Open 4 (1): 3839.

[16] Garcia, D. A., Lopes, R. D., and Hylek, E. M. 2010. "New-Onset Atrial Fibrillation and Warfarin Initiation: High Risk Periods and Implications for New Antithrombotic Drugs.” Thromb Haemost 104 (6): 1099-105. 